\title{
The interobserver reliability of the diagnosis and classification of scaphoid fractures using high- resolution peripheral quantitative CT
}

Citation for published version (APA):

Daniels, A. M., Wyers, C. E., Janzing, H. M. J., Sassen, S., Loeffen, D., Kaarsemaker, S., van Rietbergen, B., Hannemann, P. F. W., Poeze, M., \& van den Bergh, J. P. (2020). The interobserver reliability of the diagnosis and classification of scaphoid fractures using high-resolution peripheral quantitative CT. The Bone \& Joint Journal, 102B(4), 478-484. https://doi.org/10.1302/0301-620X.102B4.BJJ-2019-0632.R3

Document status and date:

Published: 01/04/2020

DOI:

10.1302/0301-620X.102B4.BJJ-2019-0632.R3

Document Version:

Publisher's PDF, also known as Version of record

Document license:

Taverne

Please check the document version of this publication:

- A submitted manuscript is the version of the article upon submission and before peer-review. There can be important differences between the submitted version and the official published version of record.

People interested in the research are advised to contact the author for the final version of the publication, or visit the DOI to the publisher's website.

- The final author version and the galley proof are versions of the publication after peer review.

- The final published version features the final layout of the paper including the volume, issue and page numbers.

Link to publication

\footnotetext{
General rights rights.

- You may freely distribute the URL identifying the publication in the public portal. please follow below link for the End User Agreement:

www.umlib.nl/taverne-license

Take down policy

If you believe that this document breaches copyright please contact us at:

repository@maastrichtuniversity.nl

providing details and we will investigate your claim.
}

Copyright and moral rights for the publications made accessible in the public portal are retained by the authors and/or other copyright owners and it is a condition of accessing publications that users recognise and abide by the legal requirements associated with these

- Users may download and print one copy of any publication from the public portal for the purpose of private study or research.

- You may not further distribute the material or use it for any profit-making activity or commercial gain

If the publication is distributed under the terms of Article $25 \mathrm{fa}$ of the Dutch Copyright Act, indicated by the "Taverne" license above, 


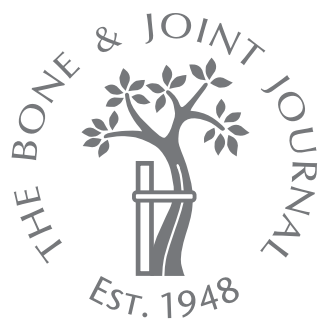

WRIST \& HAND

\title{
The interobserver reliability of the diagnosis and classification of scaphoid fractures using high-resolution peripheral quantitative CT
}
A. M. Daniels, C. E. Wyers, H. M. J. Janzing, S. Sassen, D. Loeffen, S. Kaarsemaker, B. van Rietbergen, P. F. W.
Hannemann, M. Poeze, J. P. van den Bergh

From VieCuri Medical Centre, Venlo, The Netherlands
Correspondence should be sent to A. M. Daniels; email: adaniels@viecuri.nl

(c) 2020 The British Editorial Society of Bone \& Joint Surgery doi:10.1302/0301-620X.102B4. BJJ-2019-0632.R3 $\$ 2.00$

Bone Joint $J$

2020;102-B(4):478-484.

\begin{abstract}
Aims
Besides conventional radiographs, the use of $\mathrm{MRI}, \mathrm{CT}$, and bone scintigraphy is frequent in the diagnosis of a fracture of the scaphoid. However, which techniques give the best results remain unknown. The investigation of a new imaging technique initially requires an analysis of its precision. The primary aim of this study was to investigate the interobserver agreement of high-resolution peripheral quantitative CT (HR-pOCT) in the diagnosis of a scaphoid fracture. A secondary aim was to investigate the interobserver agreement for the presence of other fractures and for the classification of scaphoid fracture.
\end{abstract}

\section{Methods}

Two radiologists and two orthopaedic trauma surgeons evaluated HR-pQCT scans of 31 patients with a clinically-suspected scaphoid fracture. The observers were asked to determine the presence of a scaphoid or other fracture and to classify the scaphoid fracture based on the Herbert classification system. Fleiss kappa statistics were used to calculate the interobserver agreement for the diagnosis of a fracture. Intraclass correlation coefficients (ICCs) were used to assess the agreement for the classification of scaphoid fracture.

\section{Results}

A total of nine $(29 \%)$ scaphoid fractures and $12(39 \%)$ other fractures were diagnosed in 20 patients $(65 \%)$ using HR-pQCT across the four observers. The interobserver agreement was $\mathbf{9 1 \%}$ for the identification of a scaphoid fracture $(95 \%$ confidence interval $(\mathrm{CI}) \mathbf{0 . 7 6}$ to 1.00 ) and $80 \%$ for other fractures $(95 \% \mathrm{Cl} 0.72$ to 0.87$)$. The mean ICC for the classification of a scaphoid fracture in the seven patients diagnosed with scaphoid fracture by all four observers was $73 \%(95 \% \mathrm{Cl} 0.42$ to 0.94$)$.

\section{Conclusion}

We conclude that the diagnosis of scaphoid and other fractures is reliable when using HRpQCT in patients with a clinically-suspected fracture.

Cite this article: Bone Joint $J$ 2020;102-B(4):478-484.

\section{Introduction}

Besides conventional radiographs, the use of MRI, $\mathrm{CT}$, and bone scintigraphy is frequent in the diagnosis of scaphoid fractures. Which technique or combination of techniques gives the best results, however, remains controversial. ${ }^{1-8}$ The lack of standard references makes the understanding of the diagnostic performance of these techniques difficult. The development of a novel low-dose radiation technique, high-resolution peripheral quantitative CT (HR-pQCT), made it possible to visualize the cortical and trabecular microarchitecture of bone. ${ }^{9,10}$ This technique has been used in many in vivo studies to assess microarchitectural changes due to ageing, osteoporosis, rheumatoid arthritis, metabolic disease, and medication. ${ }^{11-18}$ Several authors have assessed the failure load and healing process of distal radial fractures using HR-pQCT. ${ }^{19,20}$ No previous studies, however, have investigated the detection of scaphoid fractures using HR-pQCT. In order to show superior detection of scaphoid fractures an analysis of the precision of the diagnostic value of this technique and its place in the process of detection of a scaphoid fracture would be required. Thus, this study was undertaken before a comparison of HR-pQCT 


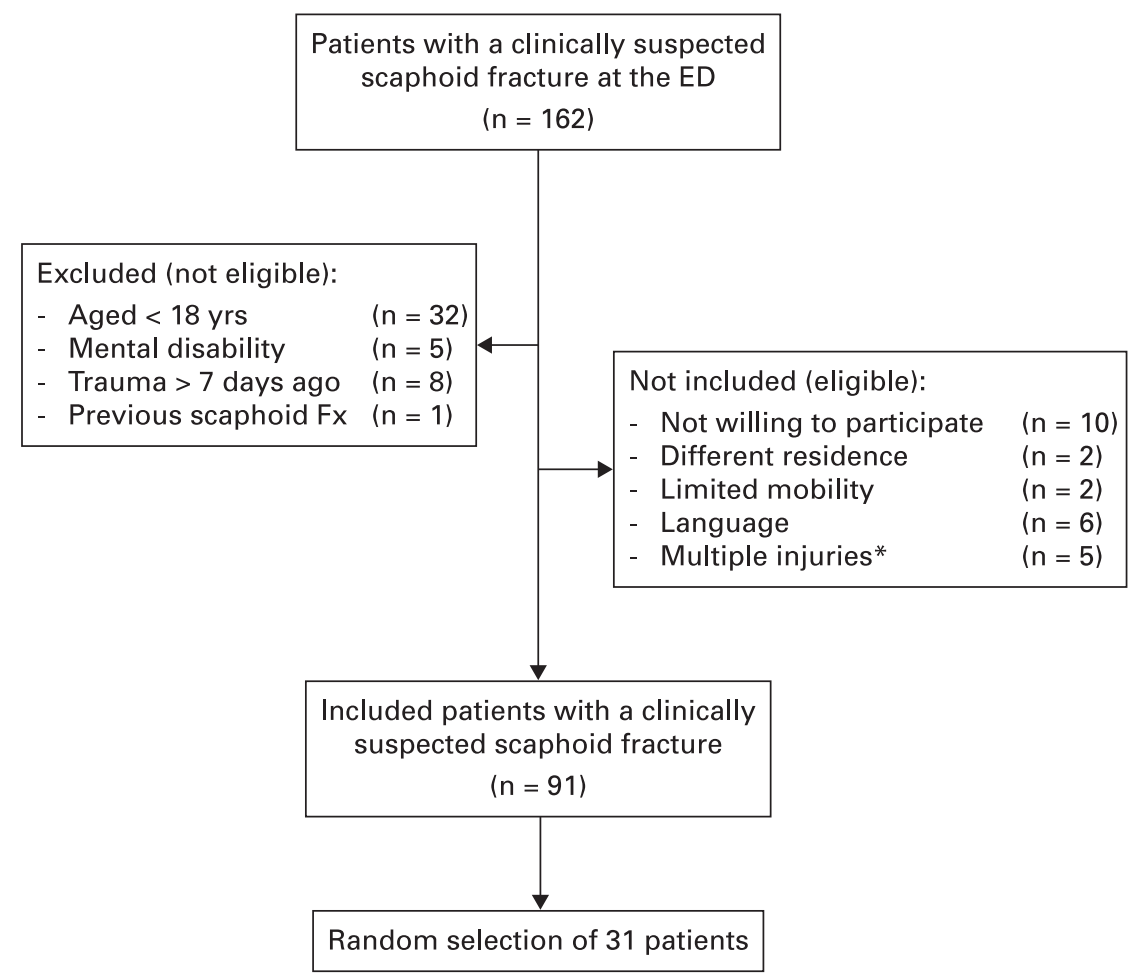

Fig. 1

Flowchart showing the eligibility criteria and selection of patients with a clinically-suspected scaphoid fracture. ${ }^{*}$ Concurrent fracture (Fx) of the ipsilateral lower/upper arm. ED, Emergency Department.

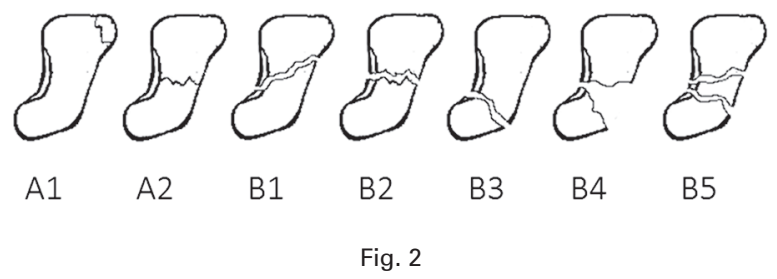

Diagram of the classification of scaphoid fracture according to Herbert and Fisher. ${ }^{33}$

with the current clinical diagnostic techniques. The primary aim was to assess the interobserver reliability of HR-pQCT scans of consecutive patients with a clinically-suspected scaphoid fracture using independent observers. A secondary aim was to assess the interobserver agreement for other fractures and for the classification of fractures of the scaphoid.

\section{Methods}

This prospective feasibility study was conducted between December 2017 and October 2018. It was approved by the Medical Ethics Committee ("SHOTGUN, Scaphoid fracture diagnosis with HR-pQCT", NL 62476.068.17), and was performed according to the principles of the Declaration of Helsinki and the Medical Research Involving Human Subjects Act (WMO).

All consecutive patients presenting to our emergency department with a suspected scaphoid fracture within one week of injury were screened for eligibility. Pregnant women and patients with a previous ipsilateral scaphoid fracture were excluded. All patients were initially treated with a forearm cast in conformity with current practice, until the time of reassessment and additional imaging. This involved 91 patients who consented and had a HR-pQCT scan of the affected forearm about ten days after presentation, as additional imaging at the time of further routine clinical evaluation. Patients with a scaphoid fracture were followed up to 26 weeks after trauma. An overview of the eligibility criteria and selection of patients is shown in Figure 1.

The sample size was determined according to the requirements described by Donner and Rotondi ${ }^{21}$ in 2010 . We aimed to achieve an interobserver agreement of $\kappa_{0}=0.80$ according to Landis and $\mathrm{Koch}^{22}$ between four observers. Based on the expected detection of a fracture using HR-pQCT, we estimated $\pi$ at 0.30 , which is slightly higher than the currently reported assessment of the incidence of $0.20 .^{2,23-27}$ The minimal acceptable limit (lower 95\% confidence interval (CI)) was prespecified as substantial agreement expressed by $\kappa_{\mathrm{L}}=0.60$, resulting in a sample size of 25 patients who needed to be examined. In order to ensure a sufficiently large cohort, we selected 31 patients using a computer-based random number generator (being one-third of our cohort) out of the 91 eligible patients. All 31 HR-pQCT scans were assessed by four independent observers.

All patients had a HR-pQCT scan using the second-generation HR-pQCT scanner (XtremeCT II; Scanco Medical AG, Zürich, Switzerland). The standard protocol of the distal radius, with an 
Table I. The characteristics of the selected $(n=31)$ and non-selected ( $n=60)$ patients.

\begin{tabular}{llll}
\hline Characteristic & $\begin{array}{l}\text { Selected } \\
\text { (n= 31) }\end{array}$ & $\begin{array}{l}\text { Non-selected } \\
\text { (n= 60) }\end{array}$ & p-value \\
\hline Median age, yrs (IOR)* & $48(29$ to 71) & $52(28$ to 65) & 0.768 \\
Male sex, $\mathrm{n}(\%)$ & $16(52)$ & $29(48)$ & 0.767 \\
$\begin{array}{l}\text { Dominant hand affected, } \\
\mathrm{n}(\%)\end{array}$ & $14(45)$ & $32(53)$ & 0.460 \\
\hline
\end{tabular}

*non-normally distributed.

$\mathrm{IQR}$, interquartile range.

isotropic voxel size of $0.061 \mathrm{~mm}$, was adapted to three consecutive stacks $(30.6 \mathrm{~mm})$ to cover the entire scaphoid. ${ }^{28-30}$ Thus the surrounding bones, including the proximal carpal row and part of the distal carpal row, were displayed. This resulted in a radiation dose of $15 \mu \mathrm{Sv}$, which is about $0.6 \%$ to $0.5 \%$ of the annual background radiation exposure for an individual in The Netherlands and USA. ${ }^{31,32}$ All scans were conducted with the wrist in a fibreglass cast with a detachable piece around the thumb, which was only used during the HR-pQCT procedure, for added stability. The forearm was placed in an anatomically shaped container to obtain a standardized position. Based on a scout view, the region of interest was determined and a reference line was placed on the rim at the articular surface of the distal radius. Motion-induced degradation of the images was graded according to the manufacturer's protocol and the method described by Pialat et al. ${ }^{30}$ Scans with motion artefacts (grade 4 to 5) were repeated once. The HR-pQCT scans were exported in Digital Imaging and Communications in Medicine (DICOM) format. The images were reconstructed into transverse, coronal, and sagittal planes of the wrist. All HR-pQCT images and reconstructions were anonymized and uploaded into a local workstation.

The independent observers were two musculoskeletal radiologists (DL, SS) and two hand and wrist trauma surgeons (HJ, SK). They were asked to determine the presence of a scaphoid or other fracture of the distal radius or (meta)carpal, and to classify the scaphoid fracture, if present, according to the Herbert and Fisher classification system ${ }^{33}$ (Figure 2). They were aware of the fact that all patients were suspected of having a scaphoid fracture clinically, were blinded to the findings on conventional radiographs and CT, and were not aware of each other's assessment. They did not have access to any clinical data.

Statistical analysis. The distribution of age was tested with Q-Q plots and the Kolmogorov-Smirnov test. Chi-squared and independent samples $t$-tests were used to analyze differences between the selected $(n=31)$ and non-selected patients $(n=60)$ using IBM SPSS Statistics (IBM, Armonk, New York, USA). Statistical significance was set at $p<0.05$. The Fleiss kappa values for the chance corrected agreement of all four observers were calculated for the presence of a scaphoid fracture and other fractures. These analyses were conducted using Microsoft Office Excel 2010 (Microsoft, Redmond, Washington, USA). The intraclass correlation coefficient (ICC) for the assessment of the classification of a scaphoid fracture was calculated with the two-way mixed model in SPSS. The interpretation of the interobserver agreement values was based on the guidelines of Landis and Koch, ${ }^{22}$ with a value between 0.0 and 0.20 representing 'slight agreement', 0.21 to 0.40 'fair agreement', 0.41 to 0.60 'moderate agreement', and 0.61 to 0.80 'substantial
Table II. Results of high-resolution peripheral quantitative CT fracture classification in 31 patients by four observers.

\begin{tabular}{|c|c|c|c|c|}
\hline \multirow[t]{2}{*}{ Patient } & \multicolumn{4}{|c|}{ Observer } \\
\hline & I & II & III & IV \\
\hline 1 & $\mathrm{O}$ & - & - & $\mathrm{O}$ \\
\hline 2 & $x$ & $X$ & $x$ & $x$ \\
\hline 3 & $x$ & $X$ & - & $x$ \\
\hline 4 & - & - & - & - \\
\hline 5 & $x$ & $X$ & $x$ & $x$ \\
\hline 6 & - & - & - & - \\
\hline 7 & $x$ & $\mathrm{X}$ & $x$ & $X$ \\
\hline 8 & $\mathrm{O}$ & $\mathrm{O}$ & - & $\mathrm{O}$ \\
\hline 9 & - & - & - & - \\
\hline 10 & - & $\mathrm{O}$ & - & $\mathrm{O}$ \\
\hline 11 & - & - & - & - \\
\hline 12 & $\mathrm{O}$ & $\mathrm{O}$ & $\mathrm{O}$ & $\mathrm{O}$ \\
\hline 13 & - & - & - & - \\
\hline 14 & $X$ & $x$ & $x$ & 0 \\
\hline 15 & $\mathrm{O}$ & $\mathrm{O}$ & $\mathrm{O}$ & 0 \\
\hline 16 & $x$ & $X$ & $x$ & $x$ \\
\hline 17 & $x$ & $x$ & $x$ & $X$ \\
\hline 18 & - & - & - & - \\
\hline 19 & $\mathrm{O}$ & $\mathrm{O}$ & 0 & 0 \\
\hline 20 & $\mathrm{O}$ & $\mathrm{O}$ & $\mathrm{O}$ & $\mathrm{O}$ \\
\hline 21 & - & - & - & - \\
\hline 22 & $X$ & $x$ & $x$ & $X$ \\
\hline 23 & $\mathrm{O}$ & $\mathrm{O}$ & $\mathrm{O}$ & $\mathrm{O}$ \\
\hline 24 & - & - & - & - \\
\hline 25 & - & - & - & - \\
\hline 26 & - & - & - & - \\
\hline 27 & - & $\mathrm{O}$ & - & - \\
\hline 28 & $\mathrm{O}$ & $\mathrm{O}$ & $\mathrm{O}$ & $\mathrm{O}$ \\
\hline 29 & $x$ & $x$ & $x$ & $x$ \\
\hline 30 & - & - & - & - \\
\hline 31 & 0 & $\mathrm{O}$ & 0 & 0 \\
\hline Total & & & & \\
\hline Scaphoid fractures (X) & 9 & 9 & 8 & 8 \\
\hline Other fractures $(\mathrm{O})$ & 9 & 10 & 7 & 11 \\
\hline No fractures (-) & 13 & 12 & 16 & 12 \\
\hline
\end{tabular}

*Scaphoid fractures $\kappa=0.91$ (95\% confidence interval 0.76 to 1.00 ).

†Scaphoid and other fractures $\kappa=0.84$ (95\% confidence interval 0.73 to 0.94$)$.

agreement'. A value $>0.80$ was considered to be an 'almost perfect agreement'.22,34

\section{Results}

The 31 patients had a median age of 48 years (interquartile range (IQR) 29 to 71 ) and $16(52 \%)$ were male. This was similar to the non-selected patients $(n=60)$ (Table I). We decided to include all scans, irrespective of the presence of motion artefacts, as this is comparable with using an imaging technique in a clinical situation. Moreover, the number of stacks (2/93) with grade 4 motion artefacts (grade 4) was low and there were no grade 5 stacks. Nine patients $(29 \%)$ were diagnosed with a scaphoid fracture by at least three observers (Table II), resulting in an interobserver agreement for the presence of a scaphoid fracture of $\kappa=0.91$ (95\% CI 0.76 to 1.00 ). Figures $3 \mathrm{a}$ and b show an example of HR-pQCT images displaying a scaphoid fracture detected by 


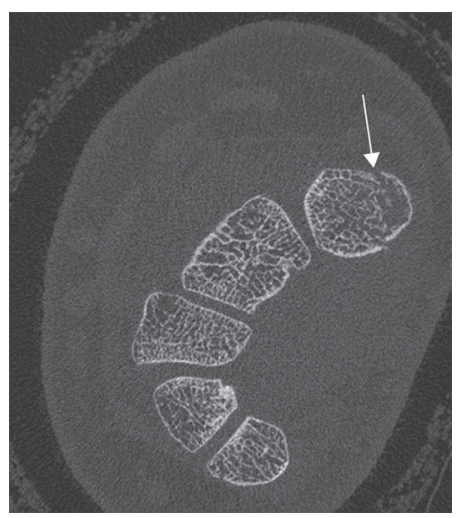

a

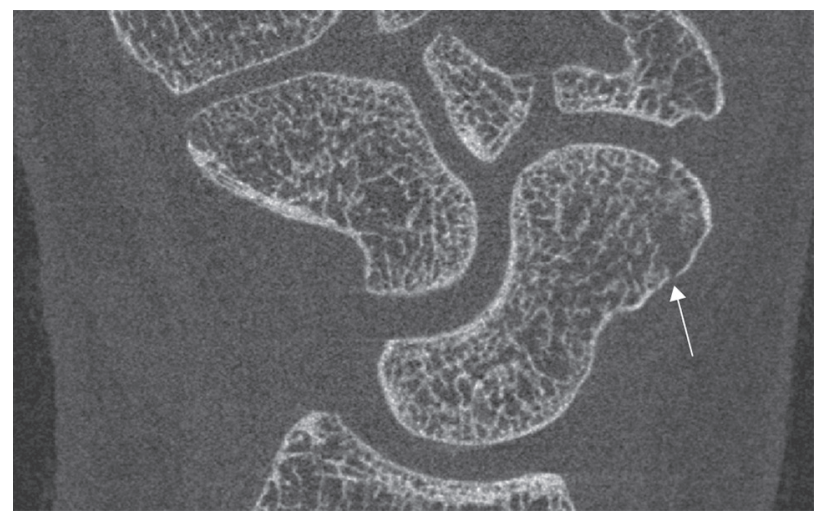

b

Fig. 3

a) Transverse high-resolution peripheral CT (HR-pQCT) section of a 48-year-old female patient with a scaphoid fracture (arrow) as diagnosed by all observers. b) Sagittal HR-pOCT section of a 48 -year-old female patient with a scaphoid fracture (arrow) as diagnosed by all observers.

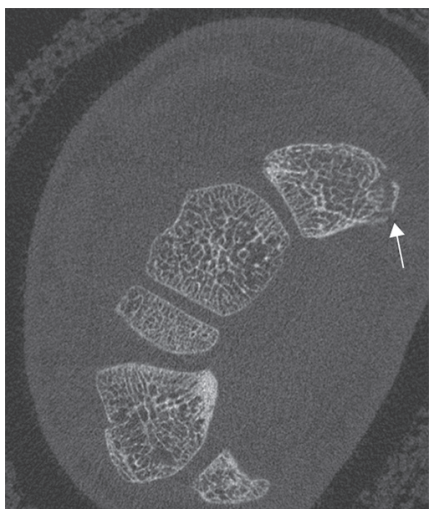

a

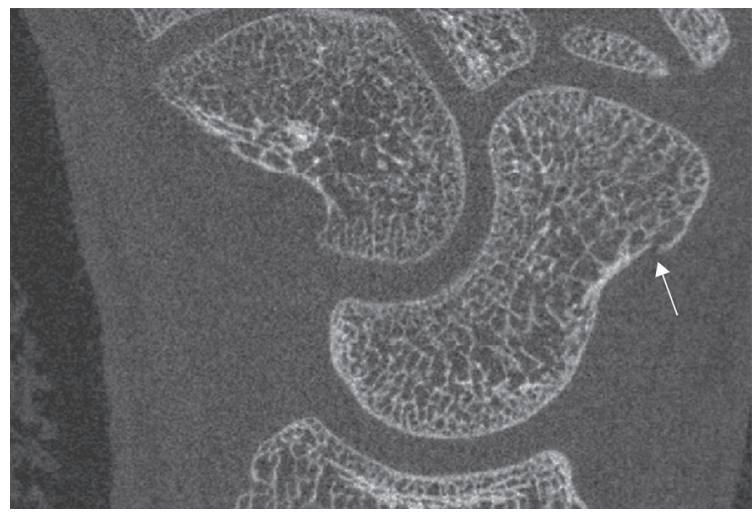

b

Fig. 4

a) Transverse high-resolution peripheral CT (HR-pQCT) section of a 18-year-old female patient with a scaphoid fracture (arrow) as diagnosed by three observers. b) Sagittal HR-pQCT section of a 18-year-old female patient with a scaphoid fracture (arrow) as diagnosed by three observers.

Table III. Herbert and Fisher ${ }^{33}$ classification of scaphoid fracture with high-resolution peripheral quantitative CT in seven patients by the four observers.*

\begin{tabular}{lllll}
\hline Patients & Observers & & & \\
\hline 1 & I & II & III & IV \\
2 & A1 & A1 & A1 & A1 \\
3 & A1 & A1 & A1 & A1 \\
4 & B1 & B2 & A2 & B1 \\
5 & A2 & B3 & B3 & B3 \\
6 & B1 & B3 & A2 & B3 \\
7 & A1 & A1 & A1 & A1 \\
Total & A1 & A1 & A1 & A1 \\
A1 & & & & \\
A2 & 4 & 4 & 4 & 4 \\
B1 & 1 & 0 & 2 & 0 \\
B2 & 2 & 0 & 0 & 1 \\
B3 & 0 & 1 & 0 & 0 \\
\hline
\end{tabular}

*Intraclass correlation coefficient $=0.73$ (95\% confidence interval 0.42 to 0.94$)$. all observers. Figures $4 a$ and $b$ show an example of images with a scaphoid fracture detected by three observers. Other fractures such as distal radial and carpal fractures were diagnosed in between seven $(23 \%)$ and $11(35 \%)$ patients by the observers (Table II). Diagnosing other fractures resulted in an interobserver agreement of $\kappa=0.80$ (95\% CI 0.72 to 0.87 ).

Seven patients $(23 \%)$ were diagnosed with a scaphoid fracture by all four observers (Table III). The ICC for the classification of a scaphoid fracture in these seven patients was 0.73 ( $95 \%$ CI 0.42 to 0.94 ), indicating substantial agreement. Figure 5 shows HR-pQCT images with a scaphoid fracture detected by all observers but classified differently according to the Herbert and Fisher ${ }^{33}$ classification system.

\section{Discussion}

We found a $90 \%$ agreement between four independent observers for the diagnosis of fracture using HR-pQCT in 31 patients with a clinically-suspected scaphoid fracture. Previous studies assessing interobserver variability in patients with a suspected 


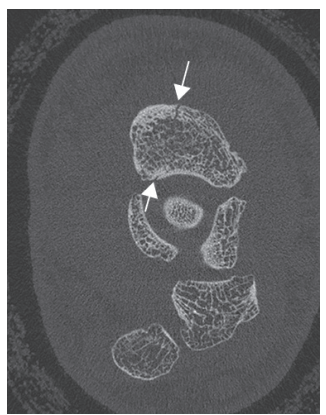

a

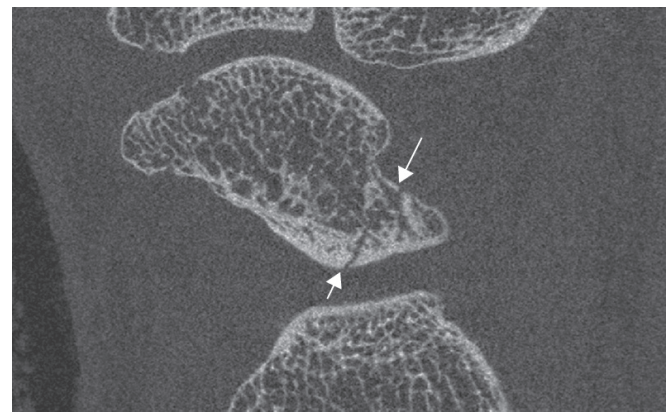

b

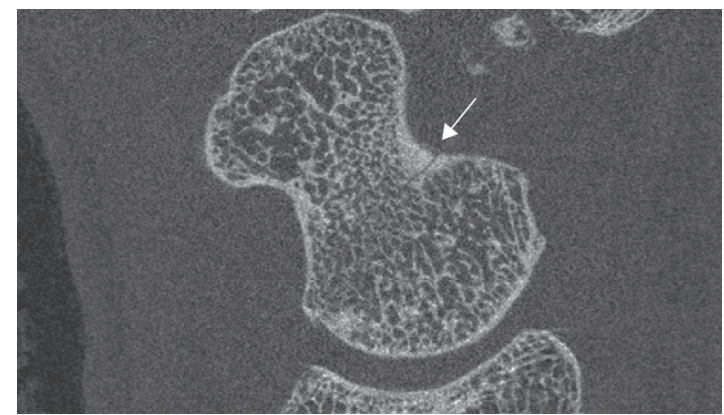

c

Fig. 5

a) Transverse HR-pQCT section of a 53-year-old male patient with a scaphoid fracture (arrow) with different classification (Herbert and Fisher ${ }^{33}$ ) by the four observers. b) Sagittal HR-pQCT section (slice of the scaphoid on the radial side) of Figure 5a. c) Sagittal HR-pOCT section (slice of the scaphoid on the ulnar side) of Figure 5a.

scaphoid fracture have been conducted for various imaging techniques. The $\kappa$-values for radiographs between two and six weeks after injury ranged from 0.14 to 0.37 , representing slight to fair agreement between observers. ${ }^{35,36}$ These rates reflect the poor sensitivity of conventional radiological assessment of scaphoid fractures. ${ }^{37-39}$ This, together with the large number of false positives, implies that follow-up radiographs cannot be considered as a reference in the diagnosis of scaphoid fractures. $^{36,38,40,41}$ Interobserver agreement for MRI assessment of scaphoid fracture by four and five observers in cohorts of 79 and 64 patients, with a similar percentage of scaphoid fractures among those suspected clinically as in our study, was $\kappa=0.67$ and $\kappa=0.44$ respectively, representing moderate to substantial agreement. ${ }^{42,43}$ Beeres et al, ${ }^{44}$ in a study in which bone scans of both wrists of 100 patients with a clinically-suspected scaphoid fracture on at least one side were analyzed by three observers, found substantial agreement, $\kappa=0.61$ to 0.80 , for the diagnosis of a scaphoid fracture and for the diagnosis of other fractures. A study addressing the interobserver agreement of CT assessment of four observers in a cohort of 150 patients with a clinically-suspected scaphoid fracture, reported a $\kappa$-value of 0.51 (moderate agreement). ${ }^{45}$ In a study by Adey et al, ${ }^{23}$ with a comparable size of cohort to ours, $30 \mathrm{CT}$ scans were assessed by eight observers. Although they used a CT with lower resolution than Beeres et $\mathrm{al},{ }^{44}$ they found a $\kappa$-value of 0.66 . As a higher number of observers should reduce the sample size of patients needed to assure a specific $\kappa$-value, our results with only four observers are promising.

Only limited data are available about the classification of a scaphoid fracture. Beeres et $\mathrm{al}^{43}$ and de Zwart et $\mathrm{al}^{45}$ assessed the interobserver variability of the location of a scaphoid fracture (proximal, middle, distal) on MRI and CT for four observers and found $\kappa$-values of 0.57 and 0.48 respectively. Interobserver agreement for the classification of a scaphoid fracture by four observers in our study was substantial ( $\kappa 0.73,95 \%$ CI 0.42 to 0.94). Although we have more than three groups, the number in each of the five groups is limited, which is an important limitation of our study. A higher number of scans should be assessed to evaluate the reliability of classification of a fracture between several observers.
The rates of agreement in our study exceed those of previous studies, suggesting that using HR-pQCT images may be reliable for the diagnosis and classification of a scaphoid fracture in patients with a clinically-suspected fracture. This might be explained by the considerable experience with other imaging techniques of the observers in our study. Moreover, as resolution increases it is conceivable that the distinction between vascular structures, motion artefacts and fractures will become clearer. Therefore, using conventional CT with higher resolution than the CT scanners used in the studies by Beeres et $\mathrm{al}^{43}$ and de Zwart et $\mathrm{al}^{45}$ might also increase interobserver agreement. However, the limited data available in the literature do not prove this hypothesis yet. Although interobserver agreement is only one aspect of diagnosis, it reveals the reliability of a technique. Another limitation of our study is that we did not determine intraobserver reliability. We did not incorporate this in our study as the agreement between different observers is more relevant for this topic and the implementation in clinical practice.

Novel techniques such as HR-pQCT should be incorporated into further studies addressing the accuracy and precision of the assessment of scaphoid fractures. Although no clear definition of a fracture on HR-pQCT is available, we found that diagnosis based on expert opinion appears to be reliable. Assessment in this study was made by both radiologists and orthopaedic trauma surgeons making extrapolation into clinical practice possible. The use of a uniform scanning protocol and DICOM viewers in this study assured that implementation and assessment in a clinical setting is achievable. The HR-pQCT scanner was designed to measure bone density and to quantify the 3D microarchitecture of distal radial and distal tibial bone (both one stack of approximately $1 \mathrm{~cm}$ ). In order to scan the scaphoid, a protocol was developed comprising three stacks, instead of one, to capture the entire bone. The total procedure time for the scaphoid is about 30 minutes, including positioning the patient and scanning. The processing and analysis takes about 30 minutes and appropriate computational hardware and software are required. This further increases the costs. At present, the HR-pQCT is mainly used in research settings and it is not ready to be used in clinical practice due to logistical considerations including the time and costs involved. The costs of 
HR-pQCT scans are not yet reimbursed by healthcare insurers. We estimate that the costs of a HR-pQCT scan are about threetimes higher than those of a routine CT scan. It is clearly likely that standard protocols for scanning the scaphoid will be developed in the near future, and that processing and analysis time will be reduced.

In conclusion, the diagnosis of scaphoid and other fractures using HR-pQCT is reliable in patients with a clinically-suspected fracture. Further research should compare the identification of a scaphoid fracture using HR-pQCT with currently used techniques in order to explore the potential of this promising new technique.

\section{Take home message}

- This study shows that HR-pQCT, a novel imaging technique, is reliable for scaphoid fracture diagnosis and classification in clinical practice.

\section{References}

1. Groves AM, Kayani I, Syed R, et al. An international survey of hospital practice in the imaging of acute scaphoid trauma. AJR Am J Roentgenol. 2006;187(6):1453-1456.

2. Mallee WH, Wang J, Poolman RW, et al. Computed tomography versus magnetic resonance imaging versus bone scintigraphy for clinically suspected scaphoid fractures in patients with negative plain radiographs. Cochrane Database Syst Rev. 2015;6:CD010023.

3. de Zwart AD, Beeres FJP, Rhemrev SJ, Bartlema K, Schipper IB. Comparison of MRI, CT and bone scintigraphy for suspected scaphoid fractures. Eur J Trauma Emerg Surg. 2016;42(6):725-731.

4. Buijze GA, Jørgsholm P, Thomsen NOB, et al. Diagnostic performance of radiographs and computed tomography for displacement and instability of acute scaphoid waist fractures. J Bone Joint Surg Am. 2012;94-A(21):1967-1974.

5. Rhemrev SJ, Ootes D, Beeres FJ, Meylaerts SA, Schipper IB. Current methods of diagnosis and treatment of scaphoid fractures. Int J Emerg Med. 2011;4:4.

6. Rhemrev SJ, de Zwart AD, Kingma LM, et al. Early computed tomography compared with bone scintigraphy in suspected scaphoid fractures. Clin Nucl Med 2010;35(12):931-934.

7. Yin Z-G, Zhang J-B, Kan S-L, Wang X-G. Diagnosing suspected scaphoid fractures: a systematic review and meta-analysis. Clin Orthop Relat Res. 2010;468(3):723-734

8. Gemme S, Tubbs R. What physical examination findings and diagnostic imaging modalities are most useful in the diagnosis of scaphoid fractures? Ann Emerg Med. 2015;65(3):308-309.

9. Link TM. Osteoporosis imaging: state of the art and advanced imaging. Radiology. 2012;263(1):3-17

10. Burghardt AJ, Krug R, Majumdar S. High-resolution imaging techniques for bone quality assessment. In: Feldman D, ed. Vitamin D. Fourth Ed. Cambridge: Academic Press, 2018:1007-1041.

11. Chapurlat RD, Laroche $\mathbf{M}$, Thomas $\mathbf{T}$, et al. Effect of oral monthly ibandronate on bone microarchitecture in women with osteopenia-a randomized placebo-controlled trial. Osteoporos Int. 2013;24(1):311-320.

12. Vilayphiou N, Boutroy S, Sornay-Rendu E, et al. Finite element analysis performed on radius and tibia HR-pQCT images and fragility fractures at all sites in postmenopausal women. Bone. 2010;46(4):1030-1037.

13. Dalzell N, Kaptoge $\mathbf{S}$, Morris $\mathbf{N}$, et al. Bone micro-architecture and determinants of strength in the radius and tibia: age-related changes in a population-based study of normal adults measured with high-resolution pQCT. Osteoporos Int 2009;20(10):1683-1694

14. Burghardt AJ, Pialat J-B, Kazakia GJ, et al. Multicenter precision of cortical and trabecular bone quality measures assessed by high-resolution peripheral quantitative computed tomography. J Bone Miner Res. 2013;28(3):524-536.

15. Khosla S, Riggs BL, Atkinson EJ, et al. Effects of sex and age on bone microstructure at the ultradistal radius: a population-based noninvasive in vivo assessment. J Bone Miner Res. 2006;21(1):124-131.

16. Okazaki N, Burghardt AJ, Chiba K, Schafer AL, Majumdar S. Bone microstructure in men assessed by HR-pQCT: associations with risk factors and differences between men with normal, low, and osteoporosis-range areal BMD. Bone Reports. 2016;5:312-319.
17. Sornay-Rendu E, Boutroy S, Duboeuf F, Chapurlat RD. Bone microarchitecture assessed by HR-pQCT as predictor of fracture risk in postmenopausal women: the OFELY study. J Bone Miner Res. 2017;32(6):1243-1251.

18. Stach CM, BÃauerle $\mathbf{M}$, Englbrecht $\mathbf{M}$, et al. Periarticular bone structure in rheumatoid arthritis patients and healthy individuals assessed by high resolution computed tomography. Arthritis Rheum. 2010;62(2):330-339.

19. de Jong JJA, Willems PC, Arts JJ, et al. Assessment of the healing process in distal radius fractures by high resolution peripheral quantitative computed tomography. Bone. 2014;64:65-74.

20. Hosseini HS, Dünki A, Fabech J, et al. Fast estimation of Colles' fracture load of the distal section of the radius by homogenized finite element analysis based on HR-pQCT. Bone. 2017;97:65-75.

21. Donner A, Rotondi MA. Sample size requirements for interval estimation of the kappa statistic for interobserver agreement studies with a binary outcome and multiple raters. Int J Biostat. 2010;6(1):31.

22. Landis JR, Koch GG. The measurement of observer agreement for categorical data. Biometrics. 1977;33(1):159-174.

23. Adey L, Souer JS, Lozano-Calderon S, et al. Computed tomography of suspected scaphoid fractures. J Hand Surg Am. 2007;32(1):61-66.

24. Ring D, Lozano-Calderón S. Imaging for suspected scaphoid fracture. J Hand Surg Am. 2008;33(6):954-957.

25. Kozin SH. Incidence, mechanism, and natural history of scaphoid fractures. Hand Clin. 2001;17(4):515-524.

26. Jenkins PJ, Slade K, Huntley JS, Robinson CM. A comparative analysis of the accuracy, diagnostic uncertainty and cost of imaging modalities in suspected scaphoid fractures. Injury. 2008;39(7):768-774.

27. Rhemrev SJ, Beeres FJP, van Leerdam RH, Hogervorst M, Ring D. Clinical prediction rule for suspected scaphoid fractures: a prospective cohort study. Injury. 2010;41(10):1026-1030.

28. Pichler W, Windisch G, Schaffler G, et al. Computer-Assisted 3-dimensional anthropometry of the scaphoid. Orthopedics. 2010;33(2):85-88.

29. Manske SL, Zhu Y, Sandino C, Boyd SK. Human trabecular bone microarchitecture can be assessed independently of density with second generation HR-pQCT. Bone. 2015;79:213-221.

30. Pialat JB, Burghardt AJ, Sode M, Link TM, Majumdar S. Visual grading of motion induced image degradation in high resolution peripheral computed tomography: impact of image quality on measures of bone density and micro-architecture. Bone. 2012;50(1):111-118.

31. No authors listed. Natural Background Sources. United States Nuclear Regulatory Commission (USNRC), 2017. https://www.nrc.gov/about-nrc/radiation/around-us/ sources/nat-bg-sources.html (date last accessed 15 January 2020).

32. Rijksinstituut voor Volksgezondheid en Milieu ANVeS. Straling in kaart, 2018. https://www.rivm.nl/straling (date last accessed 29 January 2020) [In Dutch].

33. Herbert TJ, Fisher WE. Management of the fractured scaphoid using a new bone screw. J Bone Joint Surg Br. 1984;66-B(1):114-123

34. Fleiss JL. Measuring nominal scale agreement among many raters. Psychol Bull. 1971;76(5):378-382.

35. Tiel-vanBuul MM, van BeekEJ, Borm JJJ, et al. The value of radiographs and bone scintigraphy in suspected scaphoid fracture. J Hand Surg Am. 1993;18(3):403-406.

36. Mallee WH, Mellema JJ, Guitton TG, et al. 6-Week radiographs unsuitable for diagnosis of suspected scaphoid fractures. Arch Orthop Trauma Surg. 2016;136(6):771-778

37. Ghane MR, Rezaee-Zavareh MS, Emami-Meibodi MK, Dehghani V. How trustworthy are clinical examinations and plain radiographs for diagnosis of scaphoid fractures? Trauma Mon. 2016;21(5):e23345.

38. Low G, Raby N. Can follow-up radiography for acute scaphoid fracture still be considered a valid investigation? Clin Radiol. 2005:60(10):1106-1110.

39. Balci A, Basara I, Çekdemir EY, et al. Wrist fractures: sensitivity of radiography, prevalence, and patterns in MDCT. Emerg Radiol. 2015;22(3):251-256.

40. Wijetunga AR, Tsang VH, Giuffre $\mathbf{B}$. The utility of cross-sectional imaging in the management of suspected scaphoid fractures. J Med Radiat Sci. 2019;66(1):30-37.

41. Amrami KK, Frick MA, Matsumoto JM. Imaging for acute and chronic scaphoid fractures. Hand Clin. 2019;35(3):241-257.

42. De Zwart AD, Beeres FJP, Ring D, et al. Mri as a reference standard for suspected scaphoid fractures. Br J Radiol. 2012;85(1016):1098-1101.

43. Beeres FJP, Hogervorst $\mathbf{M}$, Kingma $\mathbf{L M}$, et al. Observer variation in MRI for suspected scaphoid fractures. Br J Radiol. 2008;81(972):950-954.

44. Beeres FJP, Hogervorst M, Rhemrev SJ, et al. Reliability of bone scintigraphy for suspected scaphoid fractures. Clin Nucl Med. 2007;32(11):835-838. 
45. de Zwart AD, Beeres FJP, Kingma LM, et al. Interobserver variability among radiologists for diagnosis of scaphoid fractures by computed tomography. $J$ Hand Surg Am. 2012;37(11):2252-2256.

\section{Author information:}

A. M. Daniels, MD, MSc, Surgical Resident, Department of Surgery, ViCuri Medical Centre, Venlo, The Netherlands: NUTRIM School for Nutrition and Translational Research in Metabolism, Maastricht University, Maastricht, The Netherlands.

C. E. Wyers, PhD, MSc, Scientific Researcher, Department of Internal Medicine, Subdivision of Endocrinology, ViCuri Medical Centre, Venlo, The Netherlands; Department of Internal Medicine, Maastricht University Medical Centre, Maastricht, The Netherlands; NUTRIM School for Nutrition and Translational Research in Metabolism, Maastricht University, Maastricht, The Netherlands.

H. M. J. Janzing, MD, PhD, MSc, Trauma surgeon, Department of Surgery, ViCuri Medical Centre, Venlo, The Netherlands.

S. Sassen, MD, MS, Radiologist, Department of Radiology, ViCuri Medical Centre, Venlo, The Netherlands.

D. Loeffen, MD, MS, Radiologist, Department of Radiology, Maastricht University Medical Centre, Maastricht, The Netherlands.

S. Kaarsemaker, MD, MS, Orthopaedic Surgeon, Department of Orthopaedic Surgery, ViCuri Medical Centre, Venlo, The Netherlands.

B. van Rietbergen, PhD, MS, Professor in Orthopaedic Biomechanics, Department of Biomedical Engineering, Eindhoven University of Technology, Eindhoven, The Netherlands; Department of Orthopaedic Surgery, Research School CAPHRI, Maastricht University Medical Centre, Maastricht, The Netherlands.

P. F. W. Hannemann, MD, PhD, MS, Trauma surgeon, Department of Surgery and Trauma Surgery, Maastricht University Medical Centre, Maastricht, The Netherlands.

M. Poeze, MD, PhD, MS, Professor and Trauma Surgeon, Department of Surgery and Trauma Surgery, Maastricht University Medical Centre, Maastricht, The Netherlands; NUTRIM School for Nutrition and Translational Research in Metabolism, Maastricht University, Maastricht, The Netherlands.

J. P. van den Bergh, MD, PhD, MSc, Professor and Internist, Department of Internal Medicine, Subdivision of Endocrinology, ViCuri MedicalCentreVenlo, The Netherlands; NUTRIM School for Nutrition and
Translational Research in Metabolism, Maastricht University, Maastricht, The Netherlands; Department of Internal Medicine, Maastricht University MedicalCentreMaastricht, The Netherlands.

\section{Author contributions:}

A. M. Daniels: Conceptualized the study, Acquired the funding, Curated the data, Carried out the formal analysis, Performed the investigation, Supervised the study, Wrote the original draft, Reviewed and edited the paper.

C. E. Wyers: Conceptualized the study, Acquired the funding, Carried out the formal analysis, Supervised the study, Reviewed and edited the paper. H. M. J. Janzing: Conceptualized the study, Acquired the funding, Curated the data, Supervised the study, Reviewed and edited the paper.

S. Sassen: Curated the data, Reviewed and edited the paper.

D. Loeffen: Curated the data, Reviewed and edited the paper.

S. Kaarsemaker: Curated the data, Reviewed and edited the paper.

B. van Rietbergen: Reviewed and edited the paper.

P. F. W. Hannemann: Reviewed and edited the paper.

M. Poeze: Reviewed and edited the paper.

J. P. van den Bergh: Conceptualized the study, Acquired the funding Supervised the study, Reviewed and edited the paper.

\section{Funding statement:}

This study was supported by the VieCuri MC Trust for Innovation and Research. The author or one or more of the authors have received or will receive benefits for personal or professional use from a commercial party related indirectly to the subject of this article.

\section{ICMJE COI statement}

B.van Rietbergen reports payments for consultancy work from Scanco Medical AG, unrelated to this study.

\section{Ethical review statement}

Data for this feasibility study were extracted from our study approved by the Medical Ethics Committee ("SHOTGUN, Scaphoid fracture diagnosis with HR-pQCT", NL 62476.068.17) conducted between December 2017 and October 2018. This study was performed according to the principles of the Declaration of Helsinki and in accordance with the Medical Research Involving Human Subjects Act (WMO)

\section{Trial registration number}

NCT03899025

This article was primary edited by J. Scott. 